\title{
Razumikhin-Type Theorems in the Problem on Instability of Nonautonomous Equations with Finite Delay ${ }^{1}$
}

\author{
By \\ N. O. Sedova \\ (Ulyanovsk State University, Russia)
}

\begin{abstract}
The paper provides some theorems on complete instability of zero solution relative to a set for nonautonomous nonlinear equations with delay. The right-hand side of the equation is assumed to satisfy conditions, which provide standard existenceuniqueness-continuous dependence-continuation theory for the equation, as well as precompactness of the collection of translations in time of the right-hand side in a functional space with a metrizable compact open topology. These assumptions allow constructing limiting equations. Using conceptions of Lyapunov-Razumikhin functions and limiting equations, new instability results are obtained, which are applicable, in particular, to autonomous and periodic delayed differential equations and generalize some known results.

Key Words and Phrases. Nonlinear equations with delay, Instability, LyapunovRazumikhin functions, Limiting equations.

2000 Mathematics Subject Classification Numbers. 34K20.
\end{abstract}

\section{Introduction}

Stability of solutions of delayed differential equations has been investigated in the last few decades by numerous authors. For nonlinear equations the main tool is Lyapunov's direct method, which for functional differential equations divides into two lines: the first one employs functionals, the second one uses Lyapunov-Razumikhin functions. Razumikhin-type conditions permit the use of simple functions and allow us to circumvent the puzzle of devising a functional and computing its derivative. The idea of exploiting functions rather than functionals was found to be quite fruitful, and developed in different directions, in particular, for equations with infinite delay and Volterra equations (see, for instance, $[4,6,8,17,19,21]$ and the references therein). Moreover, Razumikhin-type conditions for the derivative are also employed to functionals (for instance, $[15,22]$ ).

Since constructing a suitable function with the prescribed properties in applications may be quite difficult, efforts are repeatedly made to weak the

1 This work is supported by Russian foundation for basic research (02-01-00877) and by Russian Federal program on support of leading scientific school researches (NSh-2000.2003.1). 
requirements imposed on Lyapunov functions. Analogs of the classic theorems are being extended in different directions. It's possible to obtain one of such extension with invariance principle for autonomous equations [7]. And for nonautonomous equations we have the method of so-called limiting equations and quasi-invariance principle [1]. The limiting equations in themselves provide much information on asymptotic behavior of the original equation's solutions $[1,5,11,14,16]$. But when coupled with Lyapunov's direct method, an examination of limiting equations' properties gives a better outcome. The results of this kind for equations with delay can be found in $[2,3,12]$.

There are not so many results in the literature regarding instability. It has been argued on occasion that instability theorems are not so useful in applications. In fact, a solution, which is unstable relative to arbitrary perturbations, may be found to be stable relative to real those. Instability theorem can assure existence in any small neighbourhood of the solution in question at least one initial point, which makes the solution leave the given neighbourhood. But we can't say what point it is and whether it is in the set of admissible perturbations. From this viewpoint, theorems ensuring complete instability or though instability relative to a set are more applicable. Actually, if for a particular equation we proved complete instability of zero solution relative to a set $S$, and intersection of a set of admissible initial points with the set $S$ is nonempty, then we can assert instability of zero solution relative to admissible perturbations.

The purpose of this paper is to provide such theorems for nonautonomous equations with delay while using ideas from [9] and results of [3]. The right-hand side of the equation is assumed to satisfy conditions, which provide standard existence-uniqueness-continuous dependence-continuation theory for the equation, as well as precompactness of the collection of translations in time of the right-hand side in some functional space with a metrizable compact open topology. These assumptions allow constructing limiting equations. Using conceptions of limiting functions and limiting equations, new instability results are obtained, which are applicable, in particular, to autonomous and periodic delayed differential equations and generalize results of [9] for such equations.

The contents of this paper are as follows. Section 2 contains basic notations and assumptions about our equation, which are employed throughout. In Sections 3 and 4 we study the complete instability of the zero solution of the equation relative to sets of two types. Some examples are used to illustrate the main results.

\section{Preliminaries}

First of all, we use the following standard notations.

Let $C(X, Y)$ be the space of continuous mappings $X \rightarrow Y, \boldsymbol{R}^{n}$ be the real $n$-vector space with a norm $|\cdot|, \boldsymbol{R}^{+}=[0,+\infty), r>0$ be a real number. We'll 
denote by $C$ the (Banach) space $C\left([-r, 0], \boldsymbol{R}^{n}\right)$ of functions $\varphi$ with the norm $\|\varphi\|=\max \{|\varphi(s)|:-r \leqslant s \leqslant 0\}$. If $x:[\alpha-r, \alpha+\beta) \rightarrow \boldsymbol{R}^{n}\left(\alpha \in \boldsymbol{R}^{+}, \beta>0\right)$ is a continuous function and $t \in[\alpha, \alpha+\beta)$, then $x_{t} \in C$ denotes the segment of $x$ at $t$ defined by the equality $x_{t}(s)=x(t+s)$ for $-r \leqslant s \leqslant 0$. If $x \in C\left([\alpha-r, \infty), \boldsymbol{R}^{n}\right)$, then the positive orbit of $x_{t}$ in $C$ is the set $\gamma^{+}\left(x_{t}\right)=\left\{x_{t}: t \geqslant \alpha\right\}$. Next, we define the positive limit set of $x_{t}$, denoted by $\omega^{+}\left(x_{t}\right)$, to be the set of all $\psi \in C$ for which there exists a sequence $t_{k} \rightarrow+\infty$ with $x_{t_{k}} \rightarrow \psi$ in $C$.

Now consider the functional differential equation with delay of the form

$$
\dot{x}(t)=X\left(t, x_{t}\right)
$$

where $X: \boldsymbol{R}^{+} \times C_{H} \rightarrow \boldsymbol{R}^{n}$ with $C_{H}=\{\varphi \in C:\|\varphi\|<H\}, 0<H \leqslant+\infty$, is a continuous functional. Assume that $|X(t, \varphi)| \leqslant m$ for $(t, \varphi) \in \boldsymbol{R}^{+} \times \bar{C}_{q}$, where $0<q<H, \quad \bar{C}_{q}=\{\varphi \in C:\|\varphi\| \leqslant q\}, \quad m=m(q)$ is a constant. Then for any initial condition $(\alpha, \varphi) \in \boldsymbol{R}^{+} \times C_{H}$ there exists a noncontinuable solution $x(t ; \alpha, \varphi)$ of (2.1) defined on $[\alpha-r, \beta), \beta>\alpha$, such that $x_{\alpha}(\alpha, \varphi)=\varphi$, and if $|x(t ; \alpha, \varphi)| \leqslant$ $q<H$ for all $t \in[\alpha-r, \beta)$ then $\beta=+\infty[10]$. Suppose that $X(t, 0) \equiv 0$ so that equation (2.1) admits the zero solution.

Let the following assumption hold:

Assumption 2.1. The functional $X(t, \varphi)$ satisfies Lipschitz condition; that is for every compact $K \subset C_{H}$ there is $l=l(K)>0$ such that for all $\varphi_{1}, \varphi_{2} \in K$ and $t \in \boldsymbol{R}^{+}$

$$
\left|X\left(t, \varphi_{2}\right)-X\left(t, \varphi_{1}\right)\right| \leqslant l\left\|\varphi_{2}-\varphi_{1}\right\|
$$

the functional $X(t, \varphi)$ is uniformly continuous with respect to $(t, \varphi) \in \boldsymbol{R}^{+} \times K$ for every compact $K \subset C_{H}$, that is for every $\varepsilon>0$ there exists $\delta=\delta(\varepsilon, K)$ such that for $\left(t_{1}, \varphi_{1}\right),\left(t_{2}, \varphi_{2}\right) \in \boldsymbol{R}^{+} \times K$ the inequalities $\left|t_{2}-t_{1}\right|<\delta,\left\|\varphi_{2}-\varphi_{1}\right\|<\delta$ imply $\left|X\left(t_{2}, \varphi_{2}\right)-X\left(t_{1}, \varphi_{1}\right)\right|<\varepsilon$.

Now assume that a sequence $\left\{q_{n}\right\}$ is such that $0<q_{1}<q_{2}<\cdots<q_{n} \rightarrow H$ as $n \rightarrow \infty$. We define for every $q_{i}$ the set $K_{i} \subset C$ of all functions $\varphi \in C$, such that for $s, s_{1}, s_{2} \in[-r, 0]$

$$
|\varphi(s)| \leqslant q_{i}, \quad\left|\varphi\left(s_{2}\right)-\varphi\left(s_{1}\right)\right| \leqslant m\left(q_{i}\right)\left|s_{2}-s_{1}\right| .
$$

Evidently, $K_{i}$ is a compact. We set $\Gamma=\bigcup_{i=1}^{\infty} K_{i}$. Note, that if $x(t ; \alpha, \varphi)$ is a solution of (2.1) defined on $[\alpha-r, \beta), \beta>\alpha+r$, then $x_{t}(\alpha, \varphi) \in \Gamma$ for $t \in[\alpha+r, \beta)$. If $\varphi \in \Gamma$ then $x_{t}(\alpha, \varphi) \in \Gamma$ for $t \in[\alpha, \beta)$. So, if we study instability, it's no difference, whether $X(t, \varphi)$ is defined on $\boldsymbol{R}^{+} \times C_{H}$ or on $\boldsymbol{R}^{+} \times \Gamma$.

Let $\mathscr{F}_{X}$ be the space of continuous functionals mapping $\boldsymbol{R}^{+} \times \Gamma$ into $\boldsymbol{R}^{n}$. By $X_{\tau}$ we mean a translation of a functional $X$ defined by the equality 
$X_{\tau}(t, \varphi)=X(t+\tau, \varphi)$. Obviously, for $X \in \mathscr{F}_{X}$ the collection of translations $\left\{X_{\tau}: \tau \in \boldsymbol{R}^{+}\right\} \subset \mathscr{F}_{X}$.

We define a convergence on $\mathscr{F}_{X}$ as the uniform one on every compact $K^{\prime} \subset \boldsymbol{R}^{+} \times \Gamma$. Namely, a sequence $\left\{X^{(n)}\right\} \subset \mathscr{F}_{X}$ converges to $X \in \mathscr{F}_{X}$ if for every $K^{\prime} \subset \boldsymbol{R}^{+} \times \Gamma$ and any $\varepsilon>0$ one has $\left|X^{(n)}(t, \varphi)-X(t, \varphi)\right|<\varepsilon$, if $n>N=$ $N\left(\varepsilon, K^{\prime}\right)$ and $(t, \varphi) \in K^{\prime}$.

Notice, that according to the construction of the domain $\Gamma$ this convergence is metrizable, see for example [1].

Under Assumption 2.1 the collection of translations $\left\{X_{\tau}(t, \varphi)=X(t+\tau, \varphi)\right.$ : $\left.\tau \in \boldsymbol{R}^{+}\right\}$is precompact in the space $\mathscr{F}_{X}$ and we can define a limiting equation for (2.1):

$$
\dot{x}(t)=X^{*}\left(t, x_{t}\right),
$$

where a functional $X^{*}: \boldsymbol{R}^{+} \times \Gamma \rightarrow \boldsymbol{R}^{n}$ is a limiting one to $X$. What this means is there exists a sequence $t_{n} \rightarrow \infty$ such that $X^{(n)}(t, \varphi)=X\left(t+t_{n}, \varphi\right) \stackrel{\mathscr{F}_{X}}{\rightarrow} X^{*}(t, \varphi)$.

Assumption 2.1 also provides the uniqueness of solution of (2.1) for an initial condition $(\alpha, \varphi) \in \boldsymbol{R}^{+} \times C_{H}$ and the uniqueness of solution of (2.3) for an initial condition $(\alpha, \varphi) \in \boldsymbol{R}^{+} \times \Gamma$. Also, if $x(t ; \alpha, \varphi)$ is a solution of (2.1) defined and bounded on $[\alpha-r,+\infty)$ (i.e., $|x(t ; \alpha, \varphi)| \leqslant q<H)$, then $\omega^{+}\left(x_{t}(\alpha, \varphi)\right)$ is nonempty, connected, compact in $C$ and contained in $\Gamma$, and $\gamma^{+}\left(x_{t}(\alpha, \varphi)\right)$ is connected, and its closure in $C$ is compact (for a proof, see, for example, [1]).

The following lemma relates solutions of (2.1) to those of (2.3):

Lemma $2.1([1])$. Let $X^{*}(t, \varphi)$ be a limiting functional to $X(t, \varphi)$ with respect to a sequence $t_{n} \rightarrow+\infty$ and sequences $\left\{\alpha_{n}\right\} \subset \boldsymbol{R}^{+},\left\{\varphi_{n}\right\} \subset \Gamma$ be such that $\alpha_{n} \rightarrow \alpha, \varphi_{n} \rightarrow \varphi$ as $n \rightarrow \infty$. Let $x\left(t ; t_{n}+\alpha_{n}, \varphi_{n}\right)$ be the solutions of (2.1), $x^{*}(t ; \alpha, \varphi)$ be the solution of (2.3) defined on $[\alpha-r, \beta)$. Then the sequence $\left\{x^{(n)}(t)=x\left(t+t_{n} ; t_{n}+\alpha_{n}, \varphi_{n}\right)\right\}$ converges to $x^{*}(t ; \alpha, \varphi)$ uniformly in $t \in[\alpha-r, \gamma]$ for any $\gamma<\beta$.

Proof. Let $X^{(n)}(t, \varphi)=X\left(t+t_{n}, \varphi\right)$. Then $x^{(n)}(t)=x\left(t+t_{n} ; t_{n}+\alpha_{n}, \varphi_{n}\right)$ is the solution of the equation $\dot{x}(t)=X^{(n)}\left(t, x_{t}\right)$. Taking into account the properties of the functional $X(t, \varphi)$ and the definition of the limiting functional $X^{*}(t, \varphi)$, it is easy to verify that the conditions of the continuous-dependence theorem [10] hold for the sequences $\left\{X^{(n)}(t, \varphi)\right\}$ and $\left\{x^{(n)}(t)\right\}$.

A function $V \in C^{1}\left(\boldsymbol{R}^{+} \times G_{H}, \boldsymbol{R}\right), G_{H}=\left\{x \in \boldsymbol{R}^{n}:|x|<H\right\}$, with $V(t, 0)=0$ is said to be a Lyapunov function. Its derivative with respect to (2.1) is a functional $V^{\prime}: \boldsymbol{R}^{+} \times C_{H} \rightarrow \boldsymbol{R}$, defined by

$$
V^{\prime}(t, \varphi)=\frac{\partial V(t, \varphi(0))}{\partial t}+\sum_{i=1}^{n} \frac{\partial V(t, \varphi(0))}{\partial x_{i}} X_{i}(t, \varphi) .
$$


We also assume that for $V$ and its partial derivatives properties below hold:

Assumption 2.2. The function $V(t, x)$ is bounded and uniformly continuous on sets of the form $\boldsymbol{R}^{+} \times \bar{G}_{q}, \bar{G}_{q}=\left\{x \in \boldsymbol{R}^{n}:|x| \leqslant q\right\}$, that is for any $q, \quad 0<q<H$, there exists $m=m(q, V)$ such that $|V(t, x)| \leqslant m$ for $(t, x) \in$ $\boldsymbol{R}^{+} \times \bar{G}_{q}$, and for every $\varepsilon>0$ there is $\delta=\delta(\varepsilon, q, V)>0$ such that for $\left(t_{1}, x_{1}\right),\left(t_{2}, x_{2}\right) \in \boldsymbol{R}^{+} \times \bar{G}_{q}$ the inequalities $\left|t_{2}-t_{1}\right|<\delta, \quad\left|x_{2}-x_{1}\right|<\delta$ imply $\left|V\left(t_{2}, x_{2}\right)-V\left(t_{1}, x_{1}\right)\right|<\varepsilon$.

Assumption 2.3. The partial derivatives

$$
\frac{\partial V}{\partial t}, \frac{\partial V}{\partial x_{1}}, \ldots, \frac{\partial V}{\partial x_{n}}
$$

are all bounded and uniformly continuous on sets of the form $\boldsymbol{R}^{+} \times \bar{G}_{q}$, $0<q<H$.

Under Assumptions 2.1-2.3 the functional $U(t, \varphi)=V^{\prime}(t, \varphi)$ is uniformly continuous and bounded on a set $\boldsymbol{R}^{+} \times K$ for every compact $K \subset C_{H}$. Hence the collections of translations $\left\{V_{\tau}(t, x)=V(t+\tau, x): \tau \in \boldsymbol{R}^{+}\right\}$and $\left\{U_{\tau}(t, \varphi)=U(t+\tau, \varphi): \tau \in \boldsymbol{R}^{+}\right\}$are precompact in corresponding spaces $\mathscr{F}_{V}=$ $C\left(\boldsymbol{R}^{+} \times \boldsymbol{R}^{n}, \boldsymbol{R}\right)$ and $\mathscr{F}_{U}=C\left(\boldsymbol{R}^{+} \times \Gamma, \boldsymbol{R}\right)$ with metrizable compact open topologies [20], [1]. So we can define a family of limiting functions to $V$, any one of which we'll denote by $V^{*}$, and a family of limiting functionals $U^{*}$.

\section{Complete instability of the zero solution}

In this section we introduce the concept of complete instability relative to a set for the zero solution of equation (2.1) with the assumptions given in the previous section. We provide several instability theorems, which are more universal, than the results of [3], [9]. We'll use the following definition for short:

Definition 3.1. Functionals $X^{*} \in \mathscr{F}_{X}, V^{*} \in \mathscr{F}_{V}, U^{*} \in \mathscr{F}_{U}$ form a limiting complex if they are limiting those to $X \in \mathscr{F}_{X}, V \in \mathscr{F}_{V}, U \in \mathscr{F}_{U}$, respectively, for the same sequence $t_{n} \rightarrow+\infty$.

Note, that for a sequence $t_{k} \rightarrow+\infty$, which defines a limiting complex $\left(X^{*}, V^{*}, U^{*}\right)$ and the limit point $\psi=\lim _{k \rightarrow \infty} x_{t_{k}}$ of the solution $x(t ; \alpha, \varphi)$, and for the solution $x^{*}(t ; 0, \psi)$ of the equation $\dot{x}(t)=X^{*}\left(t, x_{t}\right)$ we have $x_{t}^{*}(0, \psi) \in \Gamma$ for all $t \geqslant 0$ and 


$$
\begin{aligned}
U^{*}(t, & \left.x_{t}^{*}(0, \psi)\right) \\
= & \lim _{k \rightarrow \infty} U\left(t+t_{k}, x_{t+t_{k}}\left(t_{k}, x_{t_{k}}(\alpha, \varphi)\right)\right)=\lim _{k \rightarrow \infty} V^{\prime}\left(t+t_{k}, x_{t+t_{k}}(\alpha, \varphi)\right) \\
= & \lim _{k \rightarrow \infty}\left[\frac{\partial}{\partial y} V\left(t+t_{k}, x\left(t+t_{k} ; \alpha, \varphi\right)\right)\right. \\
& \left.+\sum_{i=1}^{n} \frac{\partial}{\partial x_{i}} V\left(t+t_{k}, x\left(t+t_{k} ; \alpha, \varphi\right)\right) \cdot X_{i}\left(t+t_{k}, x_{t+t_{k}}(\alpha, \varphi)\right)\right] \\
= & \lim _{k \rightarrow \infty}\left[\frac{\partial}{\partial y} V\left(t+t_{k}, x\left(t+t_{k} ; t_{k}, x_{t_{k}}(\alpha, \varphi)\right)\right)\right. \\
& \left.+\sum_{i=1}^{n} \frac{\partial}{\partial x_{i}} V\left(t+t_{k}, x\left(t+t_{k} ; t_{k}, x_{t_{k}}(\alpha, \varphi)\right)\right) \cdot X_{i}\left(t+t_{k}, x_{t+t_{k}}\left(t_{k}, x_{t_{k}}(\alpha, \varphi)\right)\right)\right]
\end{aligned}
$$

(by Lemma 2.1) $=\frac{\partial}{\partial y} V^{*}\left(t, x^{*}(t ; 0, \psi)\right)+\sum_{i=1}^{n} \frac{\partial}{\partial x_{i}} V^{*}\left(t, x^{*}(t ; 0, \psi)\right) \cdot X_{i}^{*}\left(t, x_{t}^{*}(0, \psi)\right)$

$$
=\left.\frac{\partial}{\partial s} V^{*}\left(s, x^{*}(s ; 0, \psi)\right)\right|_{s=t}
$$

(here $\left.V=V\left(y, x_{1}, \ldots, x_{n}\right)\right)$. That is,

$$
U^{*}\left(t, x_{t}^{*}(0, \psi)\right)=\left.\frac{\partial}{\partial s} V^{*}\left(s, x^{*}(s ; 0, \psi)\right)\right|_{s=t} .
$$

Let

$$
P_{M}^{t}(V)=\left\{(t, \varphi) \in \boldsymbol{R}^{+} \times C_{H}: 0<V(t, \varphi(0))=\max _{-r \leqslant s \leqslant 0} V(t+s, \varphi(s))\right\}
$$

(if $V(t, x) \equiv V(x)$ then we use the set $P_{M}(V)=\left\{\varphi \in C_{H}: 0<V(\varphi(0))=\right.$ $\left.\left.\max _{-r \leqslant s \leqslant 0} V(\varphi(s))\right\}[9]\right)$. The set $P_{M}^{t}(V) \cap\left[\boldsymbol{R}^{+} \times C_{\delta}\right]$ will always assumed to be nonempty for any $\delta \in(0, H)$.

Now we define the set

$$
N\left(U^{*}\right)=\left\{(t, \varphi) \in \boldsymbol{R}^{+} \times \Gamma: U^{*}(t, \varphi)=0\right\} .
$$

We say that the set $M \subset \boldsymbol{R}^{+} \times C_{H}$ does not contain solutions of an equation if for every solution $x(t ; \alpha, \varphi)$ of this equation there exists $t^{*} \geqslant \alpha$ such that $\left(t^{*}, x_{t^{*}}(\alpha, \varphi)\right) \notin M$.

Then, under the assumptions and the definitions given we can state our first result (its proof arises from the proof of Theorem 3.2 below, and therefore is omitted): 
Theorem 3.1. Suppose there exists a Lyapunov function $V(t, x)$ satisfying the following conditions:

1. $V^{\prime}(t, \varphi)>0$ for all $(t, \varphi) \in P_{M}^{t}(V)$;

2. there exists a sequence $t_{k} \rightarrow+\infty$ such that for the corresponding limiting complex $\left(X^{*}, V^{*}, U^{*}\right)$ the set $P_{M}^{t}\left(V^{*}\right) \cap N\left(U^{*}\right)$ does not contain solutions of the equation $\dot{x}(t)=X^{*}\left(t, x_{t}\right)$.

Then the zero solution of (2.1) is unstable.

In studies of instability for autonomous and periodic equations through the use of time-independent Lyapunov functions the authors of [9] took the following definition:

Definition 3.2. Let $D$ be a subset of $C_{H}$ with $0 \in \bar{D}$. The zero solution of (2.1) is said to be completely unstable relative to $D$ if there exists $\varepsilon>0$, such that for all $\delta \in(0, \varepsilon)$ and each $\varphi \in C_{\delta} \cap D$, there exists a $\alpha_{0} \geqslant 0$ such that for the solution $x\left(t ; \alpha_{0}, \varphi\right)$ of $(2.1)$ and some $t^{*}>\alpha_{0}$ the inequality $\left|x\left(t^{*} ; \alpha_{0}, \varphi\right)\right| \geqslant \varepsilon$ holds.

When it is considered that the right-hand side of the equation being studied depends on $t$, we change the definition in this fashion:

Definition 3.3. Let $D_{t \varphi}$ be a subset of $\boldsymbol{R}^{+} \times C_{H}, \boldsymbol{R}^{+} \times\{0\} \subset \bar{D}_{t \varphi}$. The zero solution of $(2.1)$ is said to be completely unstable relative to $D_{t \varphi}$ if there exists $\varepsilon>0$, such that for all $\delta \in(0, \varepsilon)$ and each $\varphi \in C_{\delta}, \alpha_{0} \geqslant 0$ such that $\left(\alpha_{0}, \varphi\right) \in D_{t \varphi}$ the solution $x\left(t ; \alpha_{0}, \varphi\right)$ of $(2.1)$ satisfies the inequality $\left|x\left(t^{*} ; \alpha_{0}, \varphi\right)\right| \geqslant$ $\varepsilon$ for some $t^{*}>\alpha_{0}$ (if the right-hand side $X(t, \varphi)$ is periodic in $t$ or independent on $t$, then $D_{t \varphi}=\boldsymbol{R}^{+} \times D$ with $D$ from the previous definition, but then $\alpha_{0}$ in Definition 3.2 may be an arbitrary).

Using the coined definition, we can obtain the following result:

Theorem 3.2. Let $D \subset C_{H}$ be a positively invariant set with respect to (2.1), $0 \in \bar{D}$. Suppose there exists a Lyapunov function $V(t, x)$ satisfying the following conditions:

1. the set $P_{M}^{t}(V) \cap\left[\boldsymbol{R}^{+} \times\left(D \cap C_{\delta}\right)\right]$ is nonempty for any small $\delta$;

2. $V^{\prime}(t, \varphi)>0$ for all $(t, \varphi) \in P_{M}^{t}(V) \cap\left[\boldsymbol{R}^{+} \times D\right]$;

3. there exists a sequence $t_{k} \rightarrow+\infty$ such that for the corresponding limiting complex $\left(X^{*}, V^{*}, U^{*}\right)$ the set $P_{M}^{t}\left(V^{*}\right) \cap\left[\boldsymbol{R}^{+} \times \bar{D}\right] \cap N\left(U^{*}\right)$ does not contain solutions of the equation $\dot{x}(t)=X^{*}\left(t, x_{t}\right)$.

Then the zero solution of (2.1) is completely unstable relative to $P_{M}^{t}(V) \cap$ $\left[\boldsymbol{R}^{+} \times D\right]$.

Proof. Let $\varepsilon>0$ be given, $\delta \in(0, \varepsilon)$. Let's choose $\varphi_{0}, \alpha_{0} \geqslant 0:\left\|\varphi_{0}\right\|<\delta$, $\left(\alpha_{0}, \varphi_{0}\right) \in P_{M}^{t}(V) \cap\left[\boldsymbol{R}^{+} \times D\right]$. Suppose $x(t)=x\left(t ; \alpha_{0}, \varphi_{0}\right)$ is the solution of $(2.1)$ and its norm in $\boldsymbol{R}^{n}$ is bounded by $\varepsilon$ for all $t \geqslant \alpha_{0}$. Condition 2 of the theorem 
implies the function $v(t)=V\left(t, x\left(t ; \alpha_{0}, \varphi_{0}\right)\right)$ is nondecreasing. To prove this fact, notice that $\left(\alpha_{0}, \varphi_{0}\right) \in P_{M}^{t}(V) \cap\left[\boldsymbol{R}^{+} \times D\right]$ implies $V^{\prime}\left(\alpha_{0}, \varphi_{0}\right)>0$, or $\dot{v}\left(\alpha_{0}\right)>0$. Therefore, $v(t)>v\left(\alpha_{0}\right)$ for all $t \in\left[\alpha_{0}, \alpha_{0}+\varepsilon\right], \varepsilon>0$. What this means is $\left(\alpha_{0}+\varepsilon, x_{\alpha_{0}+\varepsilon}\left(\alpha_{0}, \varphi_{0}\right)\right) \in P_{M}^{t}(V)$ and hence $V^{\prime}\left(\alpha_{0}+\varepsilon, x_{\alpha_{0}+\varepsilon}\left(\alpha_{0}, \varphi_{0}\right)\right)>0$. Replicates of similar reasoning shows that $v\left(t_{2}\right)>v\left(t_{1}\right)$ for all $t_{2}>t_{1} \geqslant \alpha_{0}$ and $\left(t, x_{t}\left(\alpha_{0}, \varphi_{0}\right)\right) \in P_{M}^{t}(V) \cap\left[\boldsymbol{R}^{+} \times D\right]$ for all $t \geqslant \alpha_{0}$. The solution in question being bounded, there is a limit

$$
\lim _{t \rightarrow+\infty} V\left(t, x\left(t ; \alpha_{0}, \varphi_{0}\right)\right)=c_{0}>V\left(\alpha_{0}, \varphi_{0}(0)\right)>0
$$

and $x_{t}\left(\alpha_{0}, \varphi_{0}\right) \in \Gamma$ for all $t \geqslant \alpha_{0}+r$. Let $t_{k} \rightarrow+\infty$ be the sequence from condition 3 of the theorem, for which $X\left(t+t_{k}, \varphi\right) \stackrel{\mathscr{F}_{X}}{\rightarrow} X^{*}(t, \varphi), V\left(t+t_{k}, x\right) \stackrel{\mathscr{F}_{V}}{\rightarrow}$ $V^{*}(t, x), \quad U\left(t+t_{k}, \varphi\right) \stackrel{\mathscr{F}_{U}}{\rightarrow} U^{*}(t, \varphi)$. The precompactness of the positive orbit $\gamma^{+}\left(x_{t}\left(\alpha_{0}, \varphi_{0}\right)\right)$ in $C$ ensures that for a subsequence $k_{j} \rightarrow \infty$ we have $x_{t_{k_{j}}}\left(\alpha_{0}, \varphi_{0}\right) \rightarrow$ $\psi \in \Gamma$. So by Lemma 2.1 the sequence $\left\{x_{j}(t)=x\left(t_{k_{j}}+t ; \alpha_{0}, \varphi_{0}\right)\right\}=\left\{x\left(t_{k_{j}}+t\right.\right.$; $\left.\left.t_{k_{j}}, x_{t_{k_{j}}}\left(\alpha_{0}, \varphi_{0}\right)\right)\right\}$ tends to the solution $x^{*}(t)=x^{*}(t ; 0, \psi)$ of $\dot{x}(t)=X^{*}\left(t, x_{t}\right)$ (uniformly in $t \in[0, T]$ for all $T>0$ ), and $x_{t}^{*} \in \Gamma$ for all $t \geqslant 0$. Since the function $V(t, x(t))$ is nondecreasing, the relation (c) yields $V^{*}\left(t, x^{*}(t ; 0, \psi)\right) \equiv c_{0}$ and $\left(t, x_{t}^{*}\right) \in P_{M}^{t}\left(V^{*}\right)$ for all $t$. Therefore by (3.1) $\left(t, x_{t}^{*}\right) \in P_{M}^{t}\left(V^{*}\right) \cap N\left(U^{*}\right)$. Furthermore, because $D$ is positively invariant, $x_{t}^{*} \in \bar{D}$ for all $t$. There arises a contradiction (see condition 3 of the theorem), so the proof is finished.

Remark 3.1. A Lyapunov function in all instability theorems presented in this paper needs not be positive definite, it is suffice that $P_{M}^{t}(V) \cap$ $\left[\boldsymbol{R}^{+} \times\left(D \cap C_{\delta}\right)\right]$ be nonempty for any small $\delta>0$.

Remark 3.2. The proof of Theorem 3.2 implies that $\varepsilon$ in Definition 3.3 may be an arbitrary (less than $H$ for $H<+\infty$ ).

The last result has a wider range of applications than Theorem 3.1, which is a corollary of Theorem 3.2 with $D=C_{H}$. Moreover, for the autonomous and periodic in $t$ equations we obtain the corollaries from the last theorem:

Theorem 3.3. Let in (2.1) $X(t, \varphi) \equiv X(\varphi), D \subset C_{H}$ be a positively invariant set with respect to (2.1), $0 \in \bar{D}$. Suppose there exists a Lyapunov function $V(x)$ satisfying the following conditions:

1. the set $D \cap P_{M}(V) \cap C_{\delta}$ is nonempty for any small $\delta>0$;

2. $V^{\prime}(\varphi)>0$ for all $\varphi \in D \cap P_{M}(V)$;

3. the set $\left\{\varphi \in \bar{D}: V^{\prime}(\varphi)=0\right\}$ does not contain solutions of the equation (2.1) except the zero solution.

Then the zero solution of $(2.1)$ is completely unstable relative to $D \cap P_{M}(V)$ (in terms of Definition 3.2) or completely unstable relative to $\boldsymbol{R}^{+} \times\left[D \cap P_{M}(V)\right]$ (in terms of Definition 3.3). 
This result is immediately evident from Theorem 3.2. It's the extension of the first statement of Theorem 3.2 [9] to the case, when a Lyapunov function can be not positive definite (and coincides with the statement of the theorem in question if $V(x)>0$ for $x \neq 0$ ). Also notice, that assertion about complete instability in terms of Definition 3.3 is more general that in terms of Definition 3.2 , since it ensures that a solution beginning in any point of the set and at any point in time will leave any preassigned neighbourhood of zero contained in $C_{H}$ (see Definition 3.3 and Remark 3.2).

Theorem 3.4. Let in (2.1) the functional $X(t, \varphi)$ be T-periodic in $t, D \subset C_{H}$ be a positively invariant set with respect to (2.1), $0 \in \bar{D}$.

Suppose there exists a T-periodic in $t$ Lyapunov function $V(t, x)$ satisfying the following conditions:

1. the set $P_{M}^{t}(V) \cap\left[\boldsymbol{R}^{+} \times\left(D \cap C_{\delta}\right)\right]$ is nonempty for any small $\delta$;

2. $V^{\prime}(t, \varphi)>0$ for all $(t, \varphi) \in P_{M}^{t}(V) \cap\left[\boldsymbol{R}^{+} \times D\right]$;

3. the set $\left\{(t, \varphi) \in \boldsymbol{R}^{+} \times \bar{D}: V^{\prime}(t, \varphi)=0\right\}$ does not contain solutions of equation (2.1) except the zero solution.

Then the zero solution of $(2.1)$ is completely unstable relative to $P_{M}^{t}(V) \cap$ $\left[\boldsymbol{R}^{+} \times D\right]$.

This assertion is evident from Theorem 3.2, when it is considered, that the right-hand side and a solution of a limiting equation, as well as functions, limiting to $V$ and $U$, can result from the corresponding original functions through a translation in time by the same value $\tau \in[0, T)$; in particular, $t_{k}=T k$ yields $X^{*}(t, \varphi)=X(t, \varphi), V^{*}(t, x)=V(t, x), U^{*}(t, \varphi)=V^{\prime}(t, \varphi)$.

Just as Theorem 3.3 generalizes Theorem 3.2 [9], Theorem 3.4 generalizes Theorem 4.2 [9] for the periodic equation.

Also, Theorem 3.2 yields a corollary for almost periodic in $t$ equations, according to the following definition [13]:

Definition 3.4. A functional $X(t, \varphi) \in C\left(\boldsymbol{R}^{+} \times C_{H}, \boldsymbol{R}^{n}\right)$ is called uniformly almost periodic in $t$ if for any $\varepsilon>0$ and $q \in(0, H)$ there exists $l=l(\varepsilon, q)>0$ such that any segment $[a, a+l], a \in \boldsymbol{R}^{+}$, contains at least one number $\tau$ such that $|X(t+\tau, \varphi)-X(t, \varphi)|<\varepsilon$ for all $(t, \varphi) \in \boldsymbol{R}^{+} \times C_{q}$.

For a functional $X(t, \varphi)$, which is uniformly almost periodic in $t$, the collection of translations $\left\{X_{\tau}(t, \varphi)=X(t+\tau, \varphi): \tau \in \boldsymbol{R}^{+}\right\}$is precompact in the space $\mathscr{F}_{X}$, and all the limiting to $X$ functionals are uniformly almost periodic in $t[20]$.

Moreover, the following lemma holds:

Lemma 3.1. If $X(t, \varphi)$ is uniformly almost periodic in $t$, then there exists a limiting functional $X^{*}(t, \varphi) \equiv X(t, \varphi)$. 
Proof. Let $\varepsilon_{n} \rightarrow 0$ and $a_{n} \rightarrow+\infty$ be arbitrary sequences. By Definition 3.4, for every $n$ there exist $l_{n}=l_{n}\left(\varepsilon_{n}, K_{n}\right)$ and $\tau_{n} \in\left[a_{n}, a_{n}+l_{n}\right]$ such that $\left|X\left(\tau_{n}+t, \varphi\right)-X(t, \varphi)\right|<\varepsilon_{n}$ for all $(t, \varphi) \in \boldsymbol{R}^{+} \times K_{n}$. The last inequality implies that $X\left(\tau_{n}+t, \varphi\right) \rightarrow X(t, \varphi)$ uniformly in every compact $K \subset \boldsymbol{R}^{+} \times \Gamma$.

Now from Lemma 3.1 and Theorem 3.2 we obtain immediately the following statement:

Theorem 3.5. Let in (2.1) the functional $X(t, \varphi)$ be uniformly almost periodic in $t, D \subset C_{H}$ be a positively invariant set with respect to $(2.1), 0 \in \bar{D}$.

Suppose there exists a Lyapunov function $V(x)$ satisfying the following conditions:

1. the set $D \cap P_{M}(V) \cap C_{\delta}$ is nonempty for any small $\delta$;

2. $V^{\prime}(t, \varphi)>0$ for all $(t, \varphi) \in \boldsymbol{R}^{+} \times\left[D \cap P_{M}(V)\right]$;

3. the set $\left\{(t, \varphi) \in \boldsymbol{R}^{+} \times \bar{D}: V^{\prime}(t, \varphi)=0\right\}$ does not contain solutions of equation (2.1) except the zero solution.

Then the zero solution of (2.1) is completely unstable relative to $\boldsymbol{R}^{+} \times$ $\left[D \cap P_{M}(V)\right]$.

Remark 3.3. In the paper [18] an instability theorem was proven utilizing a Lyapunov function with the positive definite on $P_{M}^{t}(V)$ derivative. Besides, instability was defined in [18] as the reverse of uniform stability. Theorems 3.1-3.5 assure that even though $V^{\prime}$ can be only positive on $P_{M}^{t}(V)$, we are able to obtain a more general statement.

Remark 3.4. Evidently, in all results above it suffices to require, that all the properties of a function $V(t, x)$ hold only in some neighbourhood of the equilibrium of equation (2.1), i.e.:

- the set $P_{M}^{t}(V)$ can be replaced with $P_{M}^{t}(V, B)=\{(t, \varphi):\|\varphi\| \leqslant B, 0<$ $\left.V(t, \varphi(0))=\max _{-r \leqslant s \leqslant 0} V(t+s, \varphi(s))\right\}$ for $B \in(0, H)$;

- the set $D$ can be replaced with $D^{B}=\{\varphi \in D:\|\varphi\| \leqslant B\}$ (see Theorem 3.3 [9] for an autonomous equation).

The proof differs from the above one in that only we can choose any $\varepsilon \in(0, B)$.

Example 3.1. Let's consider the scalar equation

$$
\dot{x}(t)=x(t)+x\left(t-r_{1}(t)\right)-x(t) x\left(t-r_{2}(t)\right) G\left(t, x_{t}\right),
$$

with $0<r_{i}(t) \leqslant r, G(t, \varphi): \boldsymbol{R}^{+} \times C_{H} \rightarrow \boldsymbol{R}$ are uniformly continuous functions, $g_{0} \leqslant G(t, \varphi) \leqslant G_{0}$. The set $D=\{\varphi: \varphi(s)>0, s \in[-r, 0]\}$ is positively invariant relative to equation (3.2). In fact, if $x(\cdot)$ is a solution with $x(t)=0$, $x(t+s)>0$ for $s \in[-r, 0)$, then the equation yields $\dot{x}(t)>0$ which is a contradiction. So, for $\varphi>0$ the solution $x(t ; \alpha, \varphi)>0$ for all $t \geqslant \alpha-r$. 
Let $V(x)=x$, then

$$
\begin{aligned}
V^{\prime}(t, \varphi) & =\varphi(0)+\varphi\left(-r_{1}(t)\right)-\varphi(0) \varphi\left(-r_{2}(t)\right) G(t, \varphi) \\
& =\varphi(0)+\varphi\left(-r_{1}(t)\right)-\varphi(0) \varphi\left(-r_{2}(t)\right) G_{+}(t, \varphi)-\varphi(0) \varphi\left(-r_{2}(t)\right) G_{-}(t, \varphi),
\end{aligned}
$$

where

$$
G_{-}(t, \varphi)=\left\{\begin{array}{ll}
0, & G(t, \varphi)>0 ; \\
G(t, \varphi), & G(t, \varphi) \leqslant 0 ;
\end{array} \quad G_{+}(t, \varphi)= \begin{cases}G(t, \varphi), & G(t, \varphi) \geqslant 0 \\
0, & G(t, \varphi)<0\end{cases}\right.
$$

Now, for all $\varphi \in D \cap P_{M}(V)=\{\varphi: 0<\varphi(s) \leqslant \varphi(0)\}$

$$
V^{\prime}(t, \varphi) \geqslant \varphi(0)-\varphi^{2}(0) G_{+}(t, \varphi)=\varphi(0)\left(1-\varphi(0) G_{+}(t, \varphi)\right)
$$

Therefore, $V^{\prime}(t, \varphi)>0$ for $\varphi: 0<\varphi(0)<1 / G_{0}$, if $G_{0}>0$, and for all $\varphi: \varphi(0)>$ 0 , if $G_{0} \leqslant 0$.

A limiting equation to (3.2) has the form

$$
\dot{x}(t)=x(t)+x\left(t-r_{1}^{*}(t)\right)-x(t) x\left(t-r_{2}^{*}(t)\right) G^{*}\left(t, x_{t}\right),
$$

with $r_{i}^{*}(t)=\lim _{k \rightarrow \infty} r_{i}\left(t_{k}+t\right) \quad(i=1,2), G^{*}(t, \varphi)=\lim _{k \rightarrow \infty} G\left(t_{k}+t, \varphi\right)$ for some sequence $t_{k} \rightarrow+\infty$.

Making an estimate of the functional

$$
U^{*}(t, \varphi)=\varphi(0)+\varphi\left(-r_{1}^{*}(t)\right)-\varphi(0) \varphi\left(-r_{2}^{*}(t)\right) G^{*}(t, \varphi)
$$

by analogy with the Lyapunov function derivative, we can obtain that on the set $\boldsymbol{R}^{+} \times\left[D \cap P_{M}(V) \cap C_{1 /\left|G_{0}\right|}\right]$ it is positive, so for any limiting equation the corresponding set $\left[\boldsymbol{R}^{+} \times\left\{P_{M}\left(V, 1 /\left|G_{0}\right|\right) \cap \bar{D}\right\}\right] \cap N\left(U^{*}\right)$ does not contain its solutions. Hence it follows by Theorem 3.2 that the zero solution of (3.2) is completely unstable relative to $\boldsymbol{R}^{+} \times\left[D \cap P_{M}(V)\right]$ (see Remark 3.4).

\section{Another set in complete instability results}

Let's now consider the second statement of Theorem 3.2 [9]. It implies that in Theorem 3.3 the set $P_{M}(V)$ can be replaced with $P_{m}(V)=\left\{\varphi \in C_{H}\right.$ : $\left.V(\varphi(0))=\min _{-r \leqslant s \leqslant 0} V(\varphi(s))>0\right\}$. However the similar statement for the periodic equation is not proven and proposed by the authors as an open problem. We will establish the validity of this statement for equation (2.1) with precompact right-hand side (this case covers periodic $X(t, \varphi)$ ).

With this aim in mind we introduce notations: 


$$
\begin{gathered}
P_{m}^{t}(V)=\left\{(t, \varphi) \in \boldsymbol{R}^{+} \times C_{H}: V(t, \varphi(0))=\min _{-r \leqslant s \leqslant 0} V(t+s, \varphi(s))>0\right\}, \\
n\left(t, c_{0}, V^{*}\right)=\left\{\varphi \in C_{H}: \min _{-r \leqslant s \leqslant 0} V^{*}(t+s, \varphi(s))=c_{0}\right\}, \\
m\left(t, c_{0}, V^{*}\right)=\left\{\varphi \in n\left(t, c_{0}, V^{*}\right): V^{*}(t, \varphi(0))=c_{0}\right\} .
\end{gathered}
$$

Lemma 4.1. If for a function $V(t, x)$

$$
V^{\prime}(t, \varphi) \geqslant 0 \quad \text { for all }(t, \varphi) \in P_{m}^{t}(V)
$$

then for every $(\alpha, \varphi) \in P_{m}^{t}(V)$ the following estimation holds:

$$
v(t)=V(t, x(t ; \alpha, \varphi)) \geqslant \min _{-r \leqslant s \leqslant 0} V(\alpha+s, \varphi(s)),
$$

where $x(t ; \alpha, \varphi)$ is a solution of (2.1) defined on $[\alpha-r, \beta), t \in[\alpha, \beta)$.

Proof. Suppose that the conclusion of the lemma is not true, i.e., there exists $t_{0} \in(\alpha, \beta)$ such that $v\left(t_{0}\right)<\min _{-r \leqslant s \leqslant 0} v(\alpha+s)$. Without loss of generality we can believe that $0<v\left(t_{0}\right)<v(t)$ for $t \in\left[\alpha-r, t_{0}\right)$. Then there exists $\bar{t} \in\left(\alpha, t_{0}\right]$ such that $\dot{v}(\bar{t})=V^{\prime}\left(\bar{t}, x_{\bar{t}}(\alpha, \varphi)\right)<0$ and $0<v(\bar{t})<v(t)$ for all $t \in$ $[\alpha-r, \bar{t})$. This contradiction proves the lemma.

Now under Assumptions 2.1-2.3 we can prove the next lemma (compare with Theorem 2 [3]):

Lemma 4.2. Assume that a solution $x(t ; \alpha, \varphi)$ of $(2.1)$ is defined and bounded for all $t \geqslant \alpha-r, \quad|x(t ; \alpha, \varphi)| \leqslant q<H$, and for a function $V(t, x)$ we have $V^{\prime}(t, \varphi) \geqslant 0$ for all $(t, \varphi) \in P_{m}^{t}(V)$.

Then there exists $c_{0}=$ const such that for every point $\psi$ from the positive limit set $\omega^{+}\left(x_{t}(\alpha, \varphi)\right)$ there is a limiting complex $\left(X^{*}, V^{*}, U^{*}\right)$ such the solution $x^{*}(t ; 0, \psi)$ of the limiting equation $\dot{x}(t)=X^{*}\left(t, x_{t}\right)$ has properties $x_{t}^{*} \in$ $\omega^{+}\left(x_{t}(\alpha, \varphi)\right) \subset \Gamma$ and $x_{t}^{*} \in n\left(t, c_{0}, V^{*}\right)$ for all $t \in \boldsymbol{R}^{+}$. Furthermore, if $x_{t}^{*} \in$ $m\left(t, c_{0}, V^{*}\right)$ then $U^{*}\left(t, x_{t}^{*}\right)=0$, that is $\left(t, x_{t}^{*}\right) \in N\left(U^{*}\right)$.

Proof. Let $x(t ; \alpha, \varphi)$ be a bounded solution of equation (2.1) with $(\alpha, \varphi) \in P_{m}^{t}(V)$. Then by Lemma $4.1 \min _{-r \leqslant s \leqslant 0} V(t+s, x(t+s ; \alpha, \varphi))$ is nondecreasing and because of the boundedness of $V$ along the solution there is a limit

$$
\lim _{t \rightarrow+\infty} \min _{-r \leqslant s \leqslant 0} V(t+s, x(t+s ; \alpha, \varphi))=c_{0} .
$$

Let a point $\psi \in \omega^{+}\left(x_{t}(\alpha, \varphi)\right)$ be defined by a sequence $t_{n} \rightarrow+\infty, \quad x^{(n)}=$ $x_{t_{n}}(\alpha, \varphi) \rightarrow \psi$ as $n \rightarrow \infty$ (notice that $\left\{x^{(n)}\right\} \subset \Gamma$ ). The precompactness of the collections of translations $\left\{X_{\tau}(t, \varphi): \tau \in \boldsymbol{R}^{+}\right\},\left\{V_{\tau}(t, x): \tau \in \boldsymbol{R}^{+}\right\},\left\{U_{\tau}(t, \varphi)\right.$ : 
$\left.\tau \in \boldsymbol{R}^{+}\right\}$implies that one can extract a subsequence $n_{k} \rightarrow \infty$ such that $X_{t_{n_{k}}} \stackrel{\mathscr{F}_{X}}{\rightarrow} X^{*}, V_{t_{n_{k}}} \stackrel{\mathscr{F}_{V}}{\rightarrow} V^{*}, U_{t_{n_{k}}} \stackrel{\mathscr{F}_{U}}{\rightarrow} U^{*}$. Then by Lemma 2.1 the sequence $\left\{x^{\left(n_{k}\right)}(t)\right\}=$ $\left\{x\left(t_{n_{k}}+t ; t_{n_{k}}, x^{\left(n_{k}\right)}\right)\right\}=\left\{x\left(t_{n_{k}}+t ; \alpha, \varphi\right)\right\}$ tends to the solution $x^{*}(t ; 0, \psi)$ of $\dot{x}(t)=$ $X^{*}\left(t, x_{t}\right)$ uniformly in $t$ on interval $[0, T]$ for each $T>0$.

This implies that every point $x_{t}^{*}(0, \psi), t \in \boldsymbol{R}^{+}$, is a limiting one for the sequence $\left\{x_{t_{n_{k}}+t}(\alpha, \varphi)\right\}, k \rightarrow \infty$, and $x_{t}^{*}(0, \psi) \in \omega^{+}\left(x_{t}(\alpha, \varphi)\right) \subset \Gamma$ for all $t \in \boldsymbol{R}^{+}$. By (v1),

$$
\begin{aligned}
\lim _{t \rightarrow+\infty} & \min _{-r \leqslant s \leqslant 0} V(t+s, x(t+s ; \alpha, \varphi)) \\
= & \lim _{k \rightarrow+\infty} \min _{-r \leqslant s \leqslant 0} V\left(t_{n_{k}}+t+s, x^{\left(n_{k}\right)}(t+s)\right) \\
= & \min _{-r \leqslant s \leqslant 0} V^{*}\left(t+s, x^{*}(t+s)\right)=c_{0} .
\end{aligned}
$$

Thus $x_{t}^{*} \in n\left(t, c_{0}, V^{*}\right)$ for every $t \geqslant 0$.

Let $x_{t}^{*}$ belong to $m\left(t, c_{0}, V^{*}\right)$ for some $t \in \boldsymbol{R}^{+}$, that is

$$
V^{*}\left(t, x^{*}(t)\right)=\lim _{k \rightarrow \infty} V\left(t_{n_{k}}+t, x^{\left(n_{k}\right)}(t)\right)=c_{0} .
$$

Then from (v2) we have $V^{*}\left(t, x^{*}(t)\right)=\min _{-r \leqslant s \leqslant 0} V^{*}\left(t+s, x^{*}(t+s)\right)$, so equality (3.1) implies $U^{*}\left(t, x_{t}^{*}\right)=0$. This completes the proof of the lemma.

In the next theorem we say that a set $M \subset \boldsymbol{R}^{+} \times C_{H}$ does not contain solutions of an equation if for every solution $x(t ; \alpha, \varphi)$ of this equation there exists $t^{*} \geqslant \alpha$ such that $\left(t, x_{t}(\alpha, \varphi)\right) \notin M$ for all $t \in\left[t^{*}, t^{*}+r\right]$.

This change of the traditional definition (see page 5 ) is concerned with the fact that the minimum value of the function $V$ on every interval with length $r$, not the function $V$ itself, is asymptotically constant along a solution. So for any bounded solution $x^{*}(t)$ of a limiting equation the relation $\left(t, x_{t}^{*}\right) \in N\left(U^{*}\right)$ holds necessary only at some moment $t$ on every interval with length $r$, but not for all $t$. However, in many examples this change is insignificant.

Theorem 4.1. Let $D$ be a positively invariant set with respect to (2.1), $0 \in \bar{D}$. Suppose there exists a Lyapunov function $V(t, x)$ satisfying the following conditions:

1. the set $P_{m}^{t}(V) \cap\left[\boldsymbol{R}^{+} \times\left(D \cap C_{\delta}\right)\right]$ is nonempty for any small $\delta$;

2. $V^{\prime}(t, \varphi) \geqslant 0$ for all $(t, \varphi) \in P_{m}^{t}(V) \cap\left[\boldsymbol{R}^{+} \times D\right]$;

3. there exists a limiting complex $\left(X^{*}, V^{*}, U^{*}\right)$ such that the set $P_{m}^{t}\left(V^{*}\right) \cap$ $\left[\boldsymbol{R}^{+} \times \bar{D}\right] \cap N\left(U^{*}\right)$ does not contain solutions of the equation $\dot{x}(t)=$ $X^{*}\left(t, x_{t}\right)$.

Then the zero solution of $(2.1)$ is completely unstable relative to $P_{m}^{t}(V) \cap$ $\left[\boldsymbol{R}^{+} \times D\right]$. 
Proof. Let $\varepsilon>0$ be given, $\delta \in(0, \varepsilon)$. We take any $\varphi_{0}, \alpha_{0} \geqslant 0:\left\|\varphi_{0}\right\|<\delta$, $\left(\alpha_{0}, \varphi_{0}\right) \in P_{m}^{t}(V) \cap\left[\boldsymbol{R}^{+} \times D\right]$. By way of contradiction, suppose that the conclusion of the theorem is false. Then if $x(t)=x\left(t ; \alpha_{0}, \varphi_{0}\right)$ is the solution of (2.1), that (for an appropriate $\delta$ ) its norm in $\boldsymbol{R}^{n}$ must be bounded by $\varepsilon$ for all $t \geqslant \alpha_{0}$. Thus under the assumptions placed on the Lyapunov function, $v(t)=V\left(t, x\left(t ; \alpha_{0}, \varphi_{0}\right)\right)$ is bounded for all $t$, and by Lemma 4.1 $\min _{-r \leqslant s \leqslant 0} V\left(t+s, x\left(t+s ; \alpha_{0}, \varphi_{0}\right)\right)$ is a nondecreasing functional, so there is a limit

$$
\lim _{t \rightarrow+\infty} \min _{-r \leqslant s \leqslant 0} V\left(t+s, x\left(t+s ; \alpha_{0}, \varphi_{0}\right)\right)=c_{0} \geqslant V\left(\alpha_{0}, \varphi_{0}(0)\right)>0 .
$$

Let $t_{k} \rightarrow+\infty$ be the sequence from condition 3 of the theorem for which $X_{t_{k}}(t, \varphi) \stackrel{\mathscr{F}_{X}}{\rightarrow} X^{*}(t, \varphi), V_{t_{k}}(t, x) \stackrel{\mathscr{F}_{V}}{\rightarrow} V^{*}(t, x), U_{t_{k}}(t, \varphi) \stackrel{\mathscr{F}_{U}}{\rightarrow} U^{*}(t, \varphi)$. The solution $x\left(t ; \alpha_{0}, \varphi_{0}\right)$ is defined for all $t \geqslant \alpha_{0}-r$ and bounded, so there are $\psi \in \Gamma$ and a subsequence $k_{j} \rightarrow \infty$ such that $x_{t_{k_{j}}}\left(\alpha_{0}, \varphi_{0}\right) \rightarrow \psi$. Then by Lemma 2.1 the sequence $\left\{x^{\left(k_{j}\right)}(t)=x\left(t_{k_{j}}+t ; \alpha_{0}, \varphi_{0}\right)\right\}=\left\{x\left(t_{k_{j}}+t ; t_{k_{j}}, x_{t_{k_{j}}}\right)\right\}$ tends (uniformly in $[0, T]$ for any $T>0)$ to the solution $x^{*}(t)=x^{*}(t ; 0, \psi)$ of $\dot{x}(t)=X^{*}\left(t, x_{t}\right)$, and by Lemma $4.2 x_{t}^{*} \in n\left(t, c_{0}, V^{*}\right)$ for any $t \in \boldsymbol{R}^{+}$. Moreover, on every interval with length $r$ there exists a $t_{1}$ with $x_{t_{1}}^{*} \in m\left(t_{1}, c_{0}, V^{*}\right)$, and, by virtue of (c), $c_{0}>0$. Hence $\left(t_{1}, x_{t_{1}}^{*}\right) \in P_{m}^{t}\left(V^{*}\right)$, and by Lemma $4.2\left(t_{1}, x_{t_{1}}^{*}\right) \in N\left(U^{*}\right)$. In addition, $x_{t}^{*} \in \bar{D}$ for all $t$. This contradicts condition 3 of the theorem, so the proof is finished.

In particular, Theorem 4.1 is valid for autonomous, periodic and almost periodic in $t$ equations. It implies respectively the second assertion of [9, Theorem 3.2], and the statements of Theorems 3.4, 3.5, with $P_{M}^{t}(V)$ being replaced with $P_{m}^{t}(V)$ and $V^{\prime}(t, \varphi)>0$ being replaced with $V^{\prime}(t, \varphi) \geqslant 0$.

Example 4.1. Now consider the equation

$$
\dot{x}(t)=a(t) h(x(t))+\int_{-r}^{0} p(t, s) h(x(t+s)) d s,
$$

where the functions $a(t)$ and $p(t, s)$ are uniformly continuous in $t$ and bounded for all $t \in \boldsymbol{R}^{+}$and $s \in[-r, 0], p(t, s) \geqslant 0, h(x)$ is nondecreasing, $h(0)=0$, $h(x) \neq 0$ for $x \neq 0$.

We examine instability of the zero solution of this equation. Choose $V(x)=x$ as a Lyapunov function. Its derivative is

$$
V^{\prime}(t, \varphi)=a(t) h(\varphi(0))+\int_{-r}^{0} p(t, s) h(\varphi(s)) d s
$$

and on the set $P_{m}(V)$ admits the estimation: 


$$
V^{\prime}(t, \varphi) \geqslant\left(a(t)+\int_{-r}^{0} p(t, s) d s\right) h(\varphi(0))
$$

The equation limiting to (4.1) has a similar form:

$$
\dot{x}(t)=a^{*}(t) h(x(t))+\int_{-r}^{0} p^{*}(t, s) h(x(t+s)) d s,
$$

with $a^{*}(t)=\lim _{k \rightarrow \infty} a\left(t+t_{k}\right), p^{*}(t, s)=\lim _{k \rightarrow \infty} p\left(t+t_{k}, s\right)$ for some sequence $t_{k} \rightarrow+\infty$.

The corresponding functional is

$$
\begin{aligned}
U^{*}(t, \varphi) & =a^{*}(t) h(\varphi(0))+\int_{-r}^{0} p^{*}(t, s) h(\varphi(s)) d s \\
& \geqslant\left(a^{*}(t)+\int_{-r}^{0} p^{*}(t, s) d s\right) h(\varphi(0)) \quad \text { when } \varphi \in P_{m}\left(V^{*}\right) \equiv P_{m}(V)
\end{aligned}
$$

So under the conditions

$$
\begin{gathered}
a(t)+\int_{-r}^{0} p(t, s) d s>0 \\
\varlimsup_{t \rightarrow+\infty}\left(a(t)+\int_{-r}^{0} p(t, s) d s\right) \neq 0
\end{gathered}
$$

the set $\left[\boldsymbol{R}^{+} \times P_{m}\left(V^{*}\right)\right] \cap N\left(U^{*}\right)$ does not contain solutions of the corresponding limiting equation. Thus, Theorem 4.1 yields complete instability of the zero solution of (4.1) relative to $\boldsymbol{R}^{+} \times P_{m}(V)=\boldsymbol{R}^{+} \times P_{m}(x)$.

Remark 4.1. For an equation (4.1), in which $a(t) \equiv a, p(t, s) \equiv p(s)$ (or they are periodic in $t$ ), in [9] the following sufficient conditions of complete instability relative to $P_{M}\left(x^{2}\right)$ was obtained:

$$
a-\int_{-r}^{0}|p(s)| d s>0
$$

( $p(s)$ needs not be nonnegative function). In addition, the following sufficient conditions of complete instability relative to the positively invariant set $D \cap P_{M}\left(x^{2}\right)$ with $D=\{\varphi: \varphi(s) \geqslant 0, s \in[-r, 0]\}$ was presented in the form<smiles>[12NH]</smiles>

$$
a+\int_{-r}^{0} p_{-}(s) d s>0 .
$$

Clearly for $p(s)>0$ relationship $(\star)$ follows from $(\star \star)$ or $(\star \star \star)$, but not conversely. 
Remark 4.2. Let $V(x)=x^{2}$. Then the derivative of $V$ with respect to (4.1) is $V^{\prime}(t, \varphi)=a(t) h(\varphi(0)) \varphi(0)+\varphi(0) \int_{-r}^{0} p(t, s) h(\varphi(s)) d s$, and $V^{\prime}(t, \varphi) \leqslant$ $\left(a(t)+\int_{-r}^{0}|p(t, s)| d s\right) \varphi(0) h(\varphi(0))$ for $\varphi \in P_{M}(V)$. If $a(t)+\int_{-r}^{0}|p(t, s)| d s \leqslant 0$, then the zero solution of (4.1) is stable (asymptotically stable if inequality is strong and $(\star 1)$ holds, see, for instance, [3]). Therefore if $p(t, s)>0$ and inequality $(\star 1)$ is valid, then condition $(\star)$ is necessary and sufficient for complete instability of the zero solution of (4.1) relative to $\boldsymbol{R}^{+} \times P_{m}(x)$.

Example 4.2. Consider the zero solution of

$$
\dot{x}(t)=-a(t) x(t)+\int_{-r}^{0} p(t, s) x(t+s) d s,
$$

where: the functions $a(t) \geqslant 0$ and $p(t, s) \geqslant 0$ are uniformly continuous in $t$ and bounded for all $t \in \boldsymbol{R}^{+}$and $s \in[-r, 0]$;

$$
\int_{-r}^{0} p(t, s) d s \geqslant \varepsilon>0 \quad \text { for all } t \in \boldsymbol{R}^{+}
$$

the function $A(t)=\int_{0}^{t} a(\tau) d \tau$ is uniformly continuous and $\lim _{t \rightarrow+\infty} A(t)=A$.

Consider the function $V(t, x)=e^{A(t)} x$. The derivative of this function with respect to (4.2) equals

$$
\begin{aligned}
V^{\prime}(t, \varphi) & =e^{A(t)}\left(a(t) \varphi(0)+\left(-a(t) \varphi(0)+\int_{-r}^{0} p(t, s) \varphi(s) d s\right)\right) \\
& =e^{A(t)} \int_{-r}^{0} p(t, s) \varphi(s) d s .
\end{aligned}
$$

On the set $P_{m}^{t}(V)$ the inequality $e^{A(t+s)} \varphi(s) \geqslant e^{A(t)} \varphi(0)>0$ holds for all $s \in[-r, 0]$, and since $A(t)$ is nondecreasing, $\varphi(s) \geqslant e^{A(t)-A(t+s)} \varphi(0) \geqslant \varphi(0)>0$.

Hence, $V^{\prime}(t, \varphi) \geqslant e^{A(t)} \varphi(0) \int_{-r}^{0} p(t, s) d s$ on the set $P_{m}^{t}(V)$, and by virtue of conditions (4.3) $V^{\prime}(t, \varphi)>0$ for $(t, \varphi) \in P_{m}^{t}(V)$.

A limiting equation to (4.2) has the form

$$
\dot{x}(t)=\int_{-r}^{0} p^{*}(t, s) x(t+s) d s,
$$

with $p^{*}(t, s)=\lim _{k \rightarrow \infty} p\left(t+t_{k}, s\right)$ for some sequence $t_{k} \rightarrow+\infty$.

The derivative of the Lyapunov function has the corresponding limiting functional $U^{*}(t, \varphi)=e^{A} \int_{-r}^{0} p^{*}(t, s) \varphi(s) d s$, which under conditions (4.3) is evidently positive on $P_{m}^{t}\left(V^{*}\right)$. So Theorem 4.1 implies that conditions (4.3) yield the complete instability of the zero solution of (4.2) relative to $P_{m}^{t}(V)$ (compare with the instability conditions in Example 4.1). 
Example 4.3. Consider the system of two equations

$$
\left\{\begin{array}{l}
\dot{x}_{1}(t)=a(t)\left(x_{1}(t)-3 x_{2}^{2}(t)\right)+f\left(t,\left(x_{1}\right)_{t},\left(x_{2}\right)_{t}\right) x_{2}^{2}(t) ; \\
\dot{x}_{2}(t)=a(t)\left(x_{1}(t) x_{2}(t)+x_{2}^{3}(t)\right)-f\left(t,\left(x_{1}\right)_{t},\left(x_{2}\right)_{t}\right) x_{1}(t) x_{2}(t),
\end{array}\right.
$$

where $0<a_{0} \leqslant a(t) \leqslant a_{1}$ is uniformly continuous function, $f\left(t, \varphi_{1}, \varphi_{2}\right)$ meets Assumption 2.1. We take $V(x)=\left(x_{1}^{2}+x_{2}^{2}\right) / 2$ as a Lyapunov function. Its derivative with respect to (4.4) equals

$$
V^{\prime}(t, \varphi)=a(t)\left(\varphi_{1}(0)-\varphi_{2}^{2}(0)\right)^{2}
$$

and it is semidefinite. Limiting systems have the following form:

$$
\left\{\begin{array}{l}
\dot{x}_{1}(t)=a^{*}(t)\left(x_{1}(t)-3 x_{2}^{2}(t)\right)+f^{*}\left(t,\left(x_{1}\right)_{t},\left(x_{2}\right)_{t}\right) x_{2}^{2}(t) \\
\dot{x}_{2}(t)=a^{*}(t)\left(x_{1}(t) x_{2}(t)+x_{2}^{3}(t)\right)-f^{*}\left(t,\left(x_{1}\right)_{t},\left(x_{2}\right)_{t}\right) x_{1}(t) x_{2}(t)
\end{array}\right.
$$

where $a^{*}(t)=\lim _{t_{k} \rightarrow+\infty} a\left(t+t_{k}\right), f^{*}\left(t, \varphi_{1}, \varphi_{2}\right)=\lim _{t_{k} \rightarrow+\infty} f\left(t+t_{k}, \varphi_{1}, \varphi_{2}\right)$. The set $N\left(U^{*}\right)$ is equal to $\left\{(t, \varphi): \varphi_{1}(0)=\varphi_{2}^{2}(0)\right\}$. Substituting $x_{2}^{2}(t)$ for $x_{1}(t)$ in the system (4.5) one obtains $\dot{x}_{2}(t)=-x_{2}(t) \dot{x}_{1}(t)$. On the other hand, $\dot{x}_{1}(t)=$ $2 x_{2}(t) \dot{x}_{2}(t)$. Thus $\dot{x}_{1}(t)=0$. Assume that there exists $t \in \boldsymbol{R}^{+}$such that $2 a^{*}(t)-f^{*}\left(t, \varphi_{1}, \varphi_{2}\right) \neq 0$ with $\varphi_{1}(s) \equiv c^{2}$ and $\varphi_{2}(s) \equiv c$ for any $c \in \boldsymbol{R}$. Then for $x_{1}(t)=x_{2}^{2}(t)$ from the first equation of (4.5) one has $x_{2}^{2}(t)=0=x_{1}(t)$. Now we can see that Theorem 4.1 is applicable in this case. Moreover, by investigating the proof of the theorem carefully, we find that for system (4.5) the set $D \cap P_{M}(V)$ actually can be replaced with $C \backslash\{0\}$. Therefore the zero solution of (4.4) is completely unstable.

\section{Acknowledgment}

The author expresses her sincere gratitude to the anonymous referee who carefully read the manuscript, pointed out some mistakes in the original version, and made remarks leading to improvement of this paper.

\section{References}

[ 1 ] Andreev, A. S. and Khusanov, D. Kh., Limiting equations in stability problem for functional differential equation, Differentzial'nye uravneniya, 34 (1998), 435-440 (in Russian).

[2] Andreev, A., On the stability of nonautonomous functional differential equation, Nonlinear Anal., Theory, Methods \& Appl., 30 (1997), 2847-2854.

[ 3 ] Andreev, A. S. and Sedova, N. O., On the stability of nonautonomous equations with delay via limiting equations, Func. Diff. Equations (Israel), 5 (1998), 21-37.

[ 4 ] Bereketoglu, H. and Győri, I., Global asymptotic stability in nonautonomous Lotka-Volterra type system with infinite delay, Journal of Math. Anal. and Appl., 210 (1997), 279-291.

[ 5 ] Burton, T. A., Almost periodic solutions of Volterra equations and attractivity, Journal of Math. Anal. and Appl., 198 (1996), 581-599. 
[ 6 ] Grimmer, R. and Seifert, G., Stability properties of Volterra integrodifferential equations, J. Differential equations, 19 (1975), 147-166.

[ 7 ] Haddock, J. R. and Terjéki, J., Lyapunov-Razumikhin functions and an invariance principle for functional differential equations, J. Differential equations, 48 (1983), 95-122.

[8] Haddock, J. R. and Terjéki, J., On the location of positive limit sets for autonomous functional differential equations with infinite delay, J. Differential equations, 86 (1990), 1-32.

[ 9 ] Haddock, J. R. and Zhao, J., Instability for autonomous and periodic functional differential equations with finite delay, Funkcialaj Ekvacioj, 39 (1996), 553-570.

[10] Hale, J. K., Theory of Functional Differential Equations, Springer-Verlag, New York, 1977.

[11] Hino, Y., Stability properties for functional differential equations with infinite delay, Tohoku Math. J., 35 (1983), 597-605.

[12] Hornor, W. E., Invariance principles and asymptotic constancy of solutions of precompact functional differential equations, Tohoku Math. J., 42 (1990), 217-229.

[13] Ignatyev, A. O., On the asymptotic stability in functional differential equations, Proceedings of the American Math. Society, 127 (1999), 1753-1760.

[14] Kato, J., Asymptotic behavior in functional differential equations with infinite delay, Lecture Notes Math., 1017 (1982), 300-312.

[15] Kato, J., Stability problem in functional differential equations with infinite delay, Funkcial. Ekvac., 21 (1978), 63-80.

[16] Murakami, S., Perturbation theorems for functional differential equations with infinite delay via limiting equations, J. Differential equations, 59 (1985), 314-335.

[17] Parrot, M. E., The limiting behavior of solutions of infinite delay differential equations, Journal of Math. Anal. and Appl., 87 (1982), 603-627.

[18] Prasolov, A. V., On Lyapunov functions application to instability investigation of solutions of delayed systems, Vestnik LGU, 19 (1981), 116-118 (in Russian).

[19] Seifert, G., Liapunov-Razumikhin conditions for asymptotic stability in functional differential equations of Volterra type, J. Differential equations, 16 (1974), 289-297.

[20] Sell, G. R., Nonautonomous differential equations and topological dynamics. I. The basic theory, Trans. Amer. Math. Soc., 127 (1967), 214-262.

[21] Terjéki, J., On the asymptotic stability of solutions of functional differential equations, Annalea Polonici Mathematici, 36 (1979), 299-314.

[22] Wang, Z., Comparison method and stability problem for functional differential equations, Tohoku Math. J., 35 (1983), 349-356.

nuna adreso:

N. O. Sedova

Department of Mathematics and Mechanics

Institute of Mathematics, Physics and

Information Technology

Ulyanovsk State University

Leo Tolstoi str., 42

432970 Ulyanovsk

Russia

E-mail: SedovaNO@ulsu.ru

(Ricevita la 4-an de februaro, 2003)

(Reviziita la 12-an de julio, 2003) 